\title{
Red Ginseng Extract Facilitates the Early Differentiation of Human Embryonic Stem Cells into Mesendoderm Lineage
}

\author{
Yoon Young Kim, ${ }^{1}$ Seung-Yup Ku, ${ }^{1,2}$ Zev Rosenwaks, ${ }^{3}$ Hung Ching Liu, ${ }^{3}$ Sun Kyung Oh,, 2 \\ Shin Yong Moon, ${ }^{1,2}$ and Young Min $\mathrm{Choi}^{1}$ \\ ${ }^{1}$ Institute of Reproductive Medicine and Population, Medical Research Center, Seoul National University, \\ Seoul 110-810, Republic of Korea \\ ${ }^{2}$ Department of Obstetrics and Gynecology, Seoul National University, College of Medicine, Seoul 110-510, Republic of Korea \\ ${ }^{3}$ CRMI, Cornell Medical College, New York, NY 10021, USA \\ Correspondence should be addressed to Seung-Yup Ku, jyhsyk@snu.ac.kr \\ Received 16 February 2010; Revised 18 April 2010; Accepted 10 July 2010
}

Copyright (๑) 2011 Yoon Young Kim et al. This is an open access article distributed under the Creative Commons Attribution License, which permits unrestricted use, distribution, and reproduction in any medium, provided the original work is properly cited.

Human embryonic stem cells (hESCs) have capacities to self-renew and differentiate into all cell types in vitro. Red ginseng (RG) is known to have a wide range of pharmacological effects in vivo; however, the reports on its effects on hESCs are few. In this paper, we tried to demonstrate the effects of RG on the proliferation and differentiation of hESCs. Undifferentiated hESCs, embryoid bodies (EBs), and hESC-derived cardiac progenitors (CPs) were treated with RG extract at 0.125, 0.25, and 0.5 mg/mL. After treatment of undifferentiated hESCs from day 2 to day 6 of culture, BrdU labeling showed that RG treatment increased the proliferation of hESCs, and the expression of Oct4 and Nanog was increased in RG-treated group. To find out the effects of RG on early differentiation stage cells, EBs were treated with RG extract for 10 days and attached for further differentiation. Immunostaining for three germ layer markers showed that RG treatment increased the expressions of Brachyury and HNF3 $\beta$ on EBs. Also, RG treatment increased the expression of Brachyury in early-stage and of Nkx2.5 in late-stage hESC-derived CPs. These results demonstrate facilitating effects of RG extract on the proliferation and early differentiation of hESC.

\section{Introduction}

Ginseng, the root of Panax Ginseng C. A. Meyer, has been widely used in oriental medicine for over a thousand years and is popular worldwide in alternative medicine $[1,2]$. It has been known to possess a wide range of pharmacological effects including immunomodulatory, antiaging, anticancer, anti-inflammatory, antimutagenic activity and antioxidant activity [3-7]. In addition, ginseng is reported to have effects on various heart-related diseases and to have cardioprotective action [8].

Human embryonic stem cells (hESCs), which are derived from the inner cell mass of blastocyst, have capacities to self-renew indefinitely and differentiate into various types of cells in vitro $[9,10]$. Therefore, hESCs are considered as a cell source for cell-based therapy and present an ideal model for in vitro drug screening and studies of human development. However, studies related to hESCs have focused on differentiation into specific cell lineage such as neurons [11], pancreatic $\beta$ cell [12], cardiomyocytes [13], and the mechanism that regulates self-renewal or the signaling pathway for differentiation into certain cell types. Therefore, studies about the use of hESCs or derived differentiated cells as a developmental or preclinical model for the screening of molecules have been performed on a small, limited scale.

Recently, Wang et al. showed that treatment with the total saponins of Panax ginseng promoted the proliferation and differentiation into dopaminergic neurons of human embryonic neural stem cells [14]. Sasaki et al. demonstrated that treatment with a Panax ginseng compound promoted the differentiation of mouse embryonic stem cells into cardiomyocytes [15]. However, the effects of ginseng on the proliferation and differentiation of hESCs are still unknown. 
TABLe 1: Primer sequences used for RT-PCR and qPCR.

\begin{tabular}{lll}
\hline Gene & Forward & Reverse \\
\hline Pluripotency & & \\
\hline GAPDH & GGCGTTCTCTTTGGAAAGGTGTTC & GTACTCAGCGGCCAGCATCG \\
Oct 4 & GAGAACAATGAGAACCTTCAGGA & CTCGAACCACATCCTTCTCT \\
Sox 2 & GCGTACGCAAATTAAAGTCCAGA & TGCTATTCTTCGGCCAGTTG \\
Nanog & GCGTACGCAAATTAAAGTCCAGA & TGCTATTCTTCGGCCAGTTG \\
\hline Differentiation & & \\
\hline Brachyury & TAAGGTGGATCTTCAGGTAGC & CATCTCATTGGTGAGCTCCCT \\
Cardiac actin & TCTATGAGGGCTACGCTTTG & CCTGACTGGAAGGTTAGATGG \\
HNF3 $\beta$ & CTACGCCAACATGAACTCCA & GAGGTCCATGATCCACTGGT \\
Nestin & CAGCTGGCGCACCTCAAGATG & AGGGAAGTTGGGCTCAGGACTGG \\
Nkx 2.5 & CTTCAAGCCAGAGGCCTACG & CCGCCTCTGTCTTCTTCAGC \\
$\alpha \mathrm{MHC}$ & GTCATTGCTGAAACCGAGAATG & GCAAAGTACTGGATGACACGCT \\
\hline
\end{tabular}

In this study, we tried to demonstrate the effects of red ginseng extract on hESCs proliferation and early differentiation. Also, we analyzed the effects of red ginseng on the expression of cardiac related genes in hESC-derived cardiac progenitors (CPs).

\section{Materials and Methods}

2.1. Preparation of Red Ginseng Extract. Korean red ginseng extract derived from the root of Panax ginseng was provided from the Korean Ginseng Corporation (Seoul, Korea). Ginseng extract was dissolved in PBS (Invitrogen, USA) at concentration of $50 \mathrm{mg} / \mathrm{ml}$ and filtered through a $0.22 \mu \mathrm{m}$ filter (Millipore, USA). The dissolved extracts were aliquoted and stored at $-20^{\circ} \mathrm{C}$ until use. Extracts were added to hESCs and differentiating cells with various concentrations- -0.125 , 0.25 , and $0.5 \mathrm{mg} / \mathrm{ml}$.

2.2. hESC Culture and EB Formation. The human embryonic stem cell line, SNUhES3 [10], was maintained as previously reported [16]. Briefly, hESCs were cultured on mitotically inactivated STO (CRL-1503; ATCC, USA) feeder layer by mechanical dissociation. hESC culture medium consisted of DMEM/F12 (Invitrogen), 20\% knockout serum replacement (KO-SR; Invitrogen), 1\% nonessential amino acids (Invitrogen), $50 \mathrm{U} / \mathrm{ml}$ penicillin (Invitrogen), $50 \mu \mathrm{g} / \mathrm{ml}$ streptomycin (Invitrogen), $0.1 \mathrm{mM} \beta$-mercaptoethanol (Sigma, USA), and $4 \mathrm{ng} / \mathrm{ml}$ basic fibroblast growth factor (bFGF; Invitrogen).

For EB formation, undifferentiated hESCs were treated with Collagenase type IV (Invitrogen) for $30 \mathrm{~min}$ at $37^{\circ} \mathrm{C}$. Dissociated hES colonies were centrifuged and washed with PBS. Washed hES colonies were transferred and cultured in suspension for several days.

2.3. Differentiation of hESC into Cardiac Progenitors. Undifferentiated hESCs were treated with $0.025 \%$ trypsin-EDTA (Invitrogen) for $3 \mathrm{~min}$ at $37^{\circ} \mathrm{C}$. Feeder cells were gently removed and washed with $\mathrm{PBS}$ (Invitrogen). hES colonies were then treated with $100 \mathrm{ng} / \mathrm{ml}$ Activin A (R\&D Systems,
Minneapolis, MN, USA) for $24 \mathrm{hr}$ and then $10 \mathrm{ng} / \mathrm{ml} \mathrm{BMP2}$ (R\&D Systems) for another 4 days in RPMI 1640 (Invitrogen), supplemented with B27 (Invitrogen). The cytokines were removed at day 5 and medium was exchanged every other day during the differentiation period.

2.4. Measurement of hESC Colony Size. To calculate the average size of hESC colonies, clumps were replated in fresh feeder layer when subcultured. Four and six days after subculture, the diameters of 20 colonies were measured using $i$ Solution Image Capture Program (Seoul, Korea).

2.5. BrdU Labeling Analysis. Cells were labeled with $10 \mu \mathrm{M}$ 5-bromo-2-deoxyuridine (BrdU, Roche Diagnostics, Germany) for $24 \mathrm{hrs}$ to assess proliferation of RG-treated hESCs. After labeling, hESCs were detached from the feeder layer using $0.5 \mathrm{mg} / \mathrm{ml}$ dispase (Invitrogen) and detached colonies were treated with $0.25 \%$ trypsin-EDTA (Invitrogen) for $5 \mathrm{~min}$ at $37^{\circ} \mathrm{C}$ to dissociate into single cells. Single cells were fixed with $70 \% \mathrm{EtOH}$ for $30 \mathrm{~min}$ at RT and denatured with $4 \mathrm{M} \mathrm{HCl}$ (Sigma) for $10 \mathrm{~min}$ at RT. Following incubation with 3\% BSA soln. containing 0.03\% Triton X100 (Sigma), cells were treated with mouse anti-BrdU antibody (Chemicon, USA) for $50 \mathrm{~min}$ at RT. After washing twice with PBST, the cells were incubated with Alexa Fluor 488labeled donkey antimouse IgG antibody (Molecular Probes) for $40 \mathrm{~min}$ at $37^{\circ} \mathrm{C}$, and then washed three times with PBST. Next, Propidium Iodide (Sigma) was added to the cells and incubated for $30 \mathrm{~min}$ at RT. Samples were analyzed using BD FACS Calibur (BD Sciences, USA).

2.6. RT-PCR and $q P C R$. Total RNAs were extracted from the cells using Trizol (Invitrogen) according to the manufacturer's instructions. cDNAs were synthesized from $1 \mu \mathrm{g}$ of RNA using Accute RT-premix (Bioneer, Korea). Primers used for RT-PCR and qPCR are listed in Table 1. PCR was performed by 30 cycles of denaturation at $95^{\circ} \mathrm{C}$ for $30 \mathrm{sec}$, annealing at $56^{\circ} \mathrm{C}$ for $30 \mathrm{sec}$ and extension at $72^{\circ} \mathrm{C}$ for $30 \mathrm{sec}$. PCR products were visualized in $2 \%$ agarose gel. 

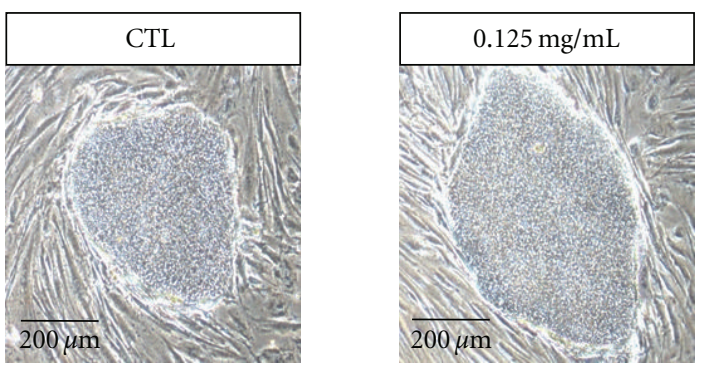
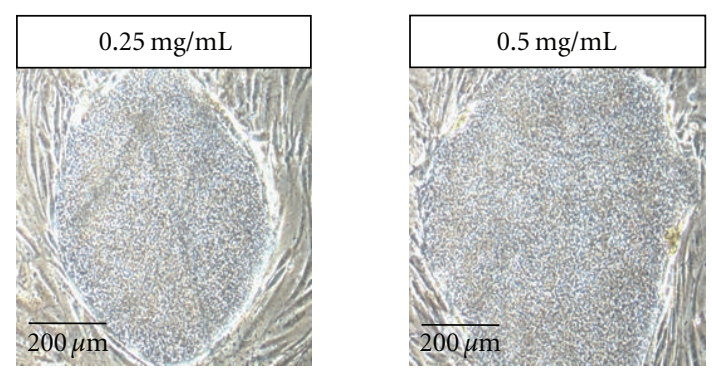

(a)

(Unit: $\mu \mathrm{m}$ )

\begin{tabular}{lcccc}
\hline & CTL & $0.125 \mathrm{mg} / \mathrm{mL}$ & $0.25 \mathrm{mg} / \mathrm{mL}$ & $0.5 \mathrm{mg} / \mathrm{mL}$ \\
\hline Day 4 hESCs & $49 \pm 13$ & $54.4 \pm 10$ & $57.8 \pm 12$ & $60 \pm 10$ \\
\hline Day 6 hESCs & $60.6 \pm 11$ & $63.1 \pm 9$ & $65 \pm 8$ & $70.9 \pm 5$ \\
\hline
\end{tabular}

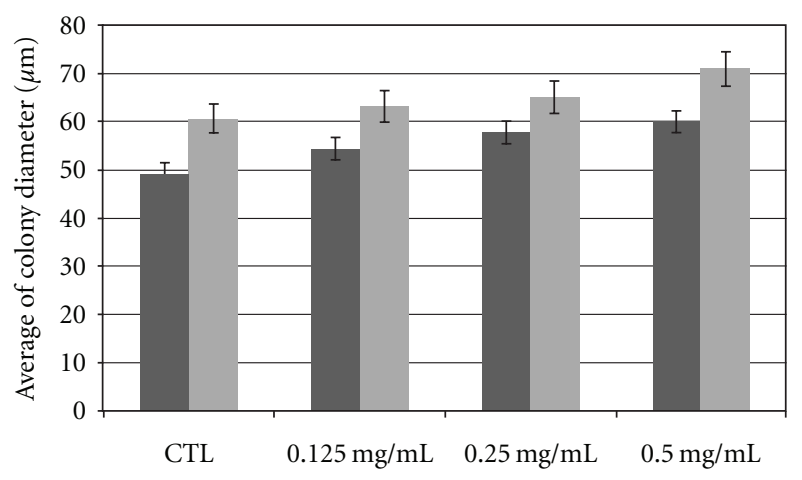

Day 4 hESCs

Day 6 hESCs

(b)

FIGURE 1: Morphological observation and measurement of colony diameter of undifferentiated hESCs after RG treatment. (a) hESCs were treated with $0,0.125,0.25$, and $0.5 \mathrm{mg} / \mathrm{ml}$ of RG extract from day 2 to day 7 . (b) Undifferentiated hESC colony diameter was calculated under the microscopic observation. Twenty colonies of each group were measured for the calculation of average diameter of hESC colony.

Quantitative PCR was carried out in RotorGene 3000 (Corbett Life Science, Sydney, Australia) using QuantiTect SYBR green PCR kit (Qiagen, Valencia, CA, USA). The amplification program included an initial step at $95^{\circ} \mathrm{C}$ for $15 \mathrm{~min}$, followed by 45 cycles of denaturation at $95^{\circ} \mathrm{C}$ for $15 \mathrm{sec}$, annealing at $58^{\circ} \mathrm{C}$ for $20 \mathrm{sec}$ and extension at $72^{\circ} \mathrm{C}$ for $30 \mathrm{sec}$. All reactions were run in triplicate. $\mathrm{C}_{\mathrm{T}}$ was calculated under default settings for the RotorGene 6.0 program (Corbett Life Science). Relative gene expression was normalized to GAPDH expression.

2.7. Immunofluorescence Staining. Samples were fixed with $4 \%$ paraformaldehyde (PFA; Sigma) for $30 \mathrm{~min}$ at RT. After washing with PBS, 3\% BSA soln. containing 0.3\% triton X100 (Sigma) was treated at $4^{\circ} \mathrm{C}$ overnight. Samples were then incubated with the primary antibodies, mouse antihuman Nestin (R\&D Systems), rabbit antihuman
Brachyury (SantaCruz Biotechnology, USA) and goat antihuman HNF3 $\beta$ (SantaCruz Biotechnology) for $1 \mathrm{hr}$ at RT. After washing with PBST, incubations with the secondary antibodies, Alexa Fluor 488-labeled donkey antigoat and rabbit IgG, Alexa Fluor 594-labeled donkey antimouse IgG, were performed for $50 \mathrm{~min}$ at RT. Samples were washed with PBST, and mounting soln. containing DAPI (Vectorshield, USA) was added. Samples were observed using a confocal laser microscope (BioRad, USA).

\section{Results}

3.1. RG Extract Treatment Enhances Proliferation of hESCs. To analyze the effects of ginseng, undifferentiated hESCs were treated with $0.125,0.25$, and $0.50 \mathrm{mg} / \mathrm{ml}$ concentrations of RG extract from day 2 to day 6. RG-treated hESC colonies were enlarged in size compared to untreated hESCs (Figure 1(a)). Average diameters of both day 4 and day 6 
CTL
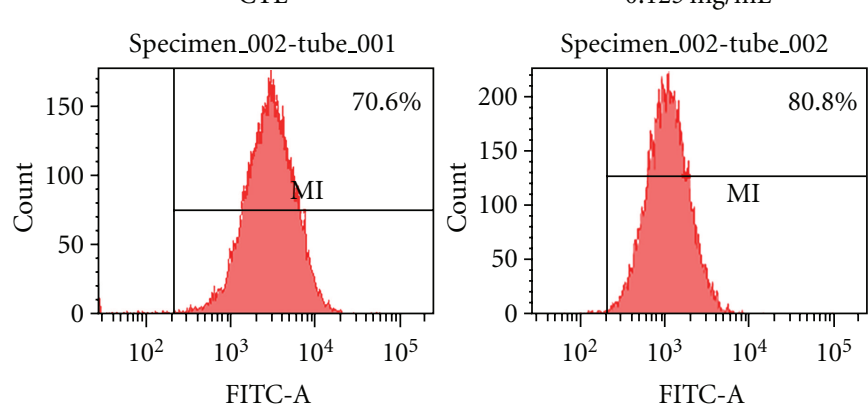

$0.25 \mathrm{mg} / \mathrm{mL}$

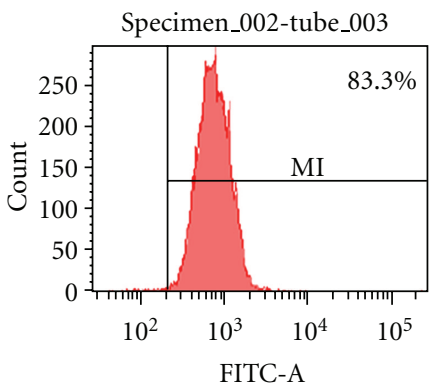

(a)

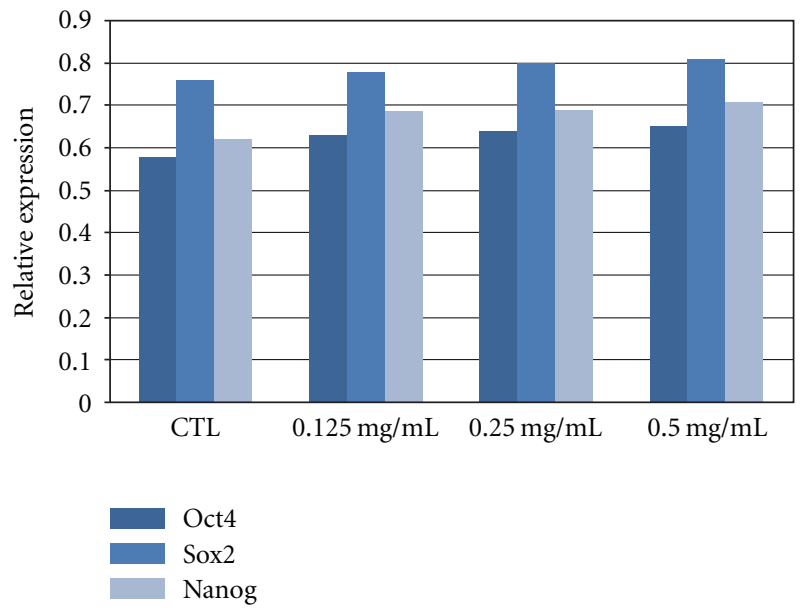

$0.5 \mathrm{mg} / \mathrm{mL}$

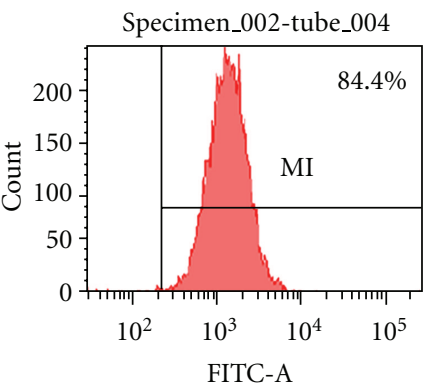

(b)

Figure 2: Proliferation and expression of pluripotency markers in RG extract-treated hESCs. (a) BrdU incorporation of RG-treated hESCs. Undifferentiated hESCs were preincubated with BrdU and analyzed by FACS. (b) Expression of stemness-related genes was evaluated by qPCR.

hESCs increased in the RG-treated group compared to the control group (Figure 1(b)). To assess the proliferative effect, the BrdU-positive population was quantified. Compared to the control, there was an increase in proliferating cells in the RG-treated group. Proportion of positive cells were $80.8 \%$,

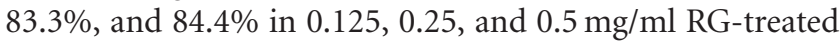
groups, respectively (Figure $2(\mathrm{a})$ ). These results showed that RG extract increased the proliferation of hESCs.

To find out the effect of RG extract on pluripotency of hESCs, the expressions of Oct4, Sox2, and Nanog were evaluated. Undifferentiated hESCs generally proliferate and express high level of pluripotency markers. Expression of Oct4 was slightly increased in the RG-treated group. Expressions of Sox 2 and Nanog were also increased in RGtreated group (Figure 2(b)). These results indicated that RG extracts also affected the expression level of pluripotency markers in undifferentiated hESCs.

\subsection{Enhanced Differentiation into Mesendoderm at Embryoid} Body Stage. To evaluate the effects of RG extract on hESCs differentiation, RG extract was added to embryoid bodies in suspension culture for 10 days (Figure 3(a), upper panel). After 10 days of suspension, EBs were reattached for further differentiation (Figure 3(a), lower panel). RT-PCR results showed that expression of Brachyury and HNF3 $\beta$ was upregulated in the RG-treated group (Figure 3(b)). Immunostaining results also showed that RG treatment increased the expression of three germ layer markers, Nestin, Brachyury, and HNF3 $\beta$, compared to the controls (Figure 3(c)). Interestingly, expressions of Brachyury and HNF3 $\beta$ were significantly upregulated in the RG-treated group. These results indicated that RG treatment of reattached EBs promoted the early phase differentiation of hESCs into mesendoderm lineage.

3.3. Upregulated Cardiac Lineage Gene Expressions in hESCDerived CPs. Effects of RG extract on cardiac specific lineage differentiation were also evaluated. Differentiated cells of day 0 to 4 (early stage) and of day 14 to 18 (late stage) were treated with RG extract. Treatment with RG significantly enhanced the expression of Brachyury and of Nkx2.5 in early stage CPs (Figure 4(a)). Also, the expression of cardiac actin, another cardiac lineage specific marker, was also increased in RGtreated early stage CPs. At day 3 and day 15, the expressions of specific proteins were examined. Brachyury, Nkx2.5, and cardiac troponin ( Tn) I showed increased expressions in the 

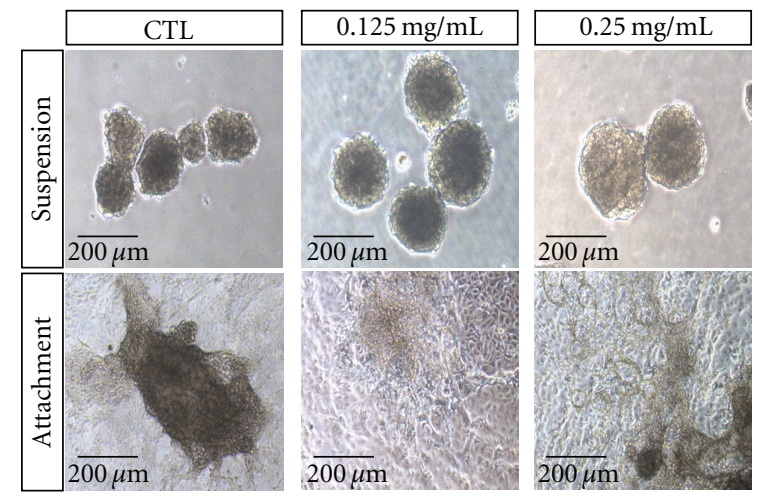

(a)

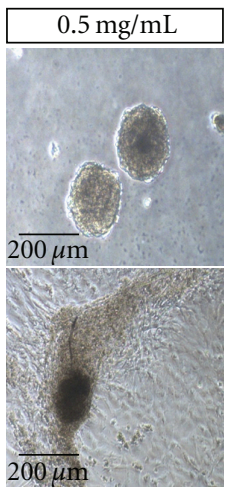

$200 \mu \mathrm{m}$

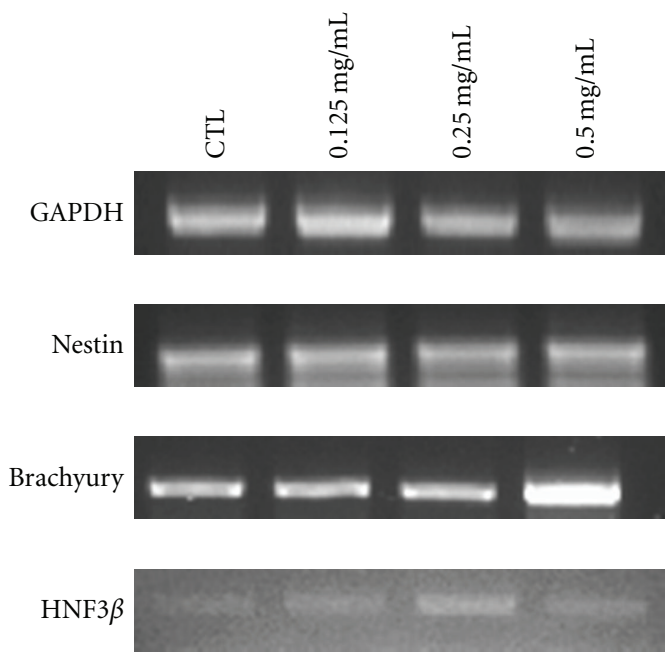

(b)

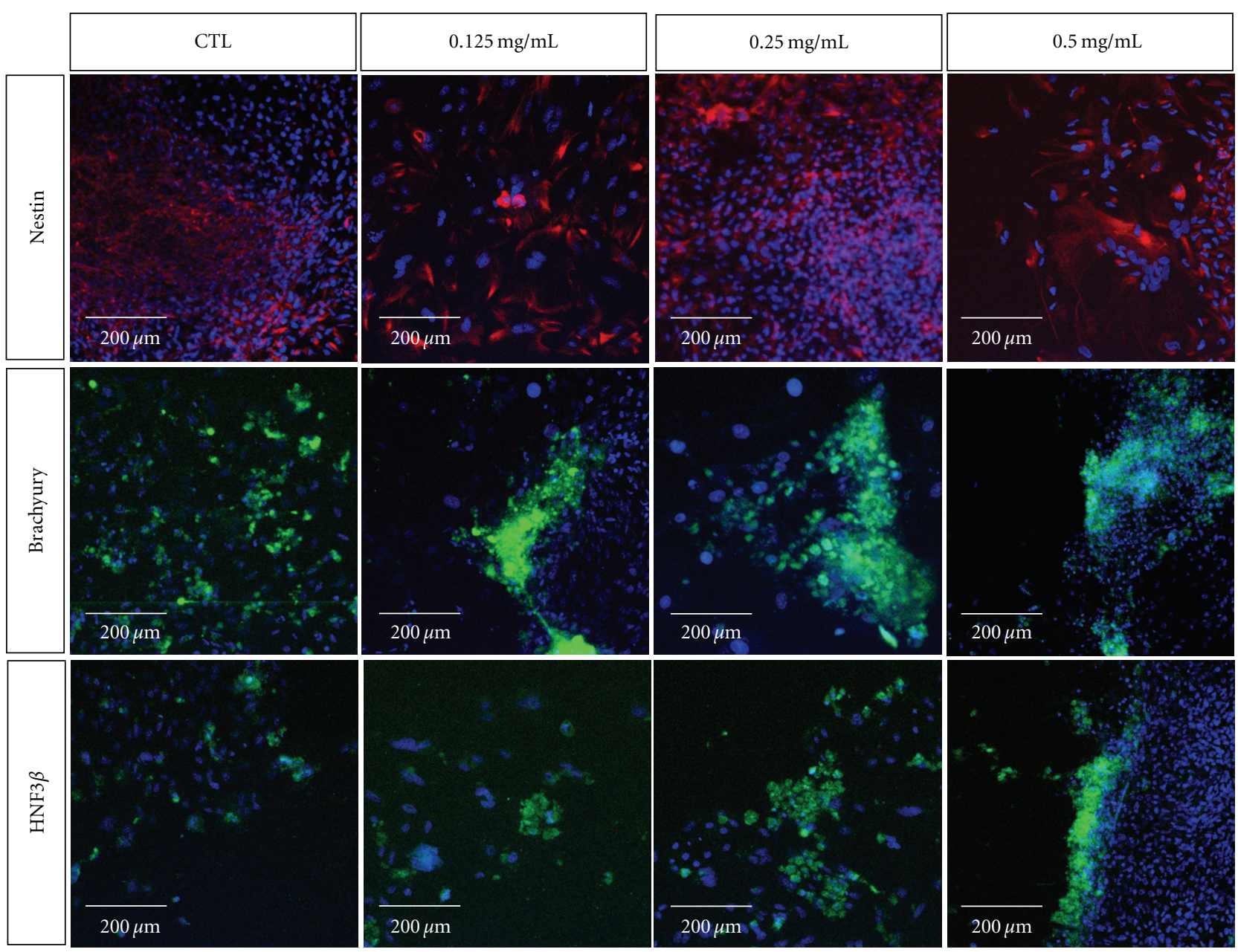

(c)

FIGURE 3: Expressions of three germ layer markers in RG-treated, differentiated cells. After 20 days of treatment, expression of early ectoderm, mesoderm, and endoderm markers were evaluated. RG-treated differentiated cells showed more dominant expression of mesodermal marker, Brachyury and endodermal marker, HNF3 $\beta$. (a) Morphological observation of differentiating cells in suspension and attached state. (b) Expressions of three germ layer markers in reattached, RG-treated cells. (c) Immunostaining results of ectoderm (Nestin), mesoderm (Brachyury) and endoderm (HNF3 $\beta)$. 

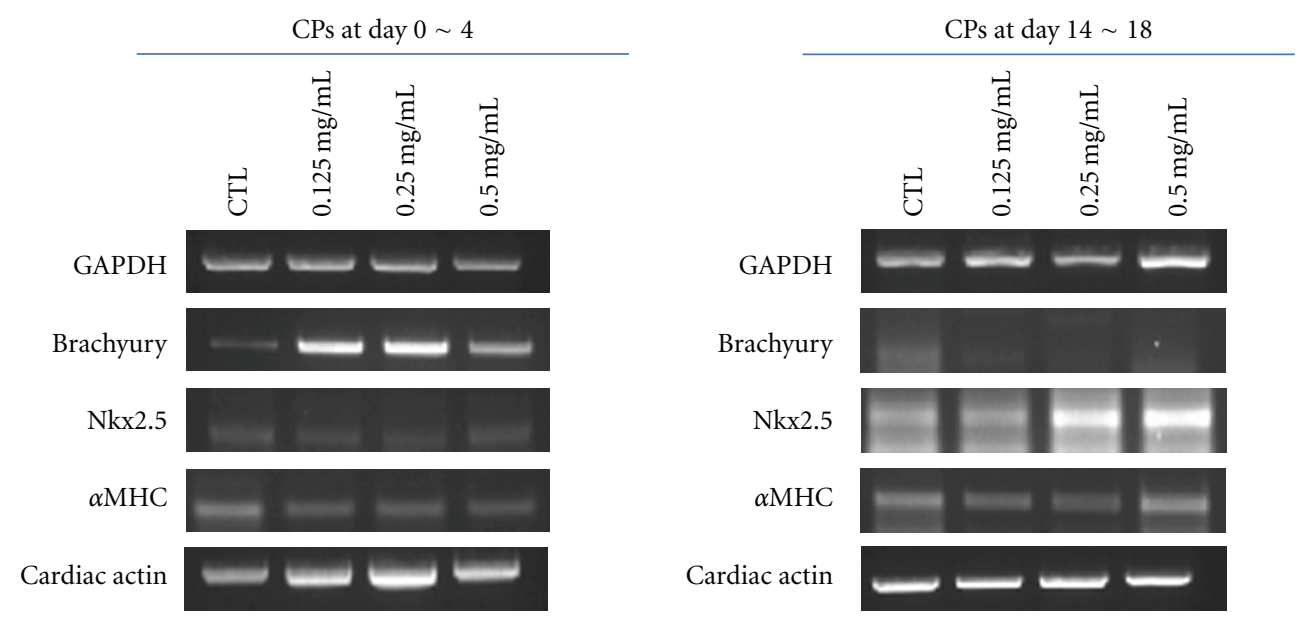

(a)
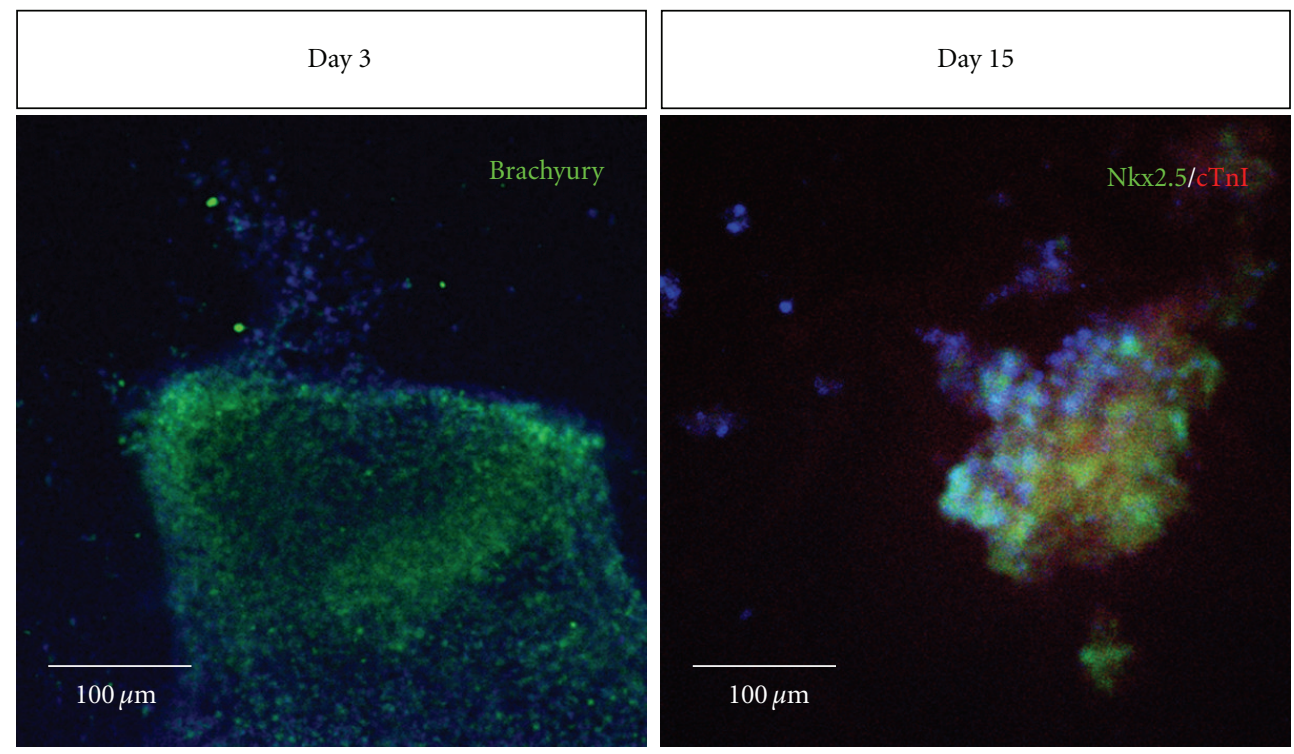

(b)

FIGURE 4: mRNA and protein expressions of mesodermal and cardiac markers in hESC-derived CPs. (a) mRNA expression of cardiac-specific markers in RG-treated, hESC-derived CPs. Expressions of Brachyury, Nkx2.5, $\alpha \mathrm{MHC}$, and cardiac actin were confirmed by RT-PCR. $1 \mu \mathrm{g}$ of RNAs were used for synthesis of cDNA. (b) Protein expression of Bachyury (early stage), Nkx2.5 and cardiac troponin I (late stage) in hESC-derived CPs.

RG-treated group (Figure 4(b)). These results demonstrated that treatment with RG extract affected the expression of cardiac-related genes and promoted the differentiation of hESC-derived differentiated cells.

\section{Discussion}

Red ginseng is a popular herbal medicine with a long history of use in Asia and has a variety of therapeutic effects [17, 18]. The main component of ginseng is ginsenoside which belongs to the steroid family named dammarane saponins $[17,19]$. Several studies have demonstrated the effect of ginseng on certain diseases using in vitro models. However, the evidence for the pharmacological effects of ginseng is still limited. Therefore, demonstrating the pharmaceutical activity of ginseng on a preclinical or clinical human model would be helpful in providing evidence for the effects of herbal medicine.

The effect of red ginseng or ginseng extract on cell proliferation or differentiation has been previously demonstrated. Zuo et al. reported that total saponins of Panax Ginseng induced K562 cells to differentiate into erythrocytes [20], and Popovich et al. showed ginseng increased viability of cultured cell [21]. In addition, Lei et al. reported that the Panax ginseng extract promoted the migration of vascular endothelial cells and expression of vascular endothelial growth factor (VEGF) mRNA [22]. Another group demonstrated that treatment with ginseng extract enhanced the contractility of mouse embryonic stem cell-derived cardiomyocytes [15]. Even with these studies, in the scientific literature to date, 


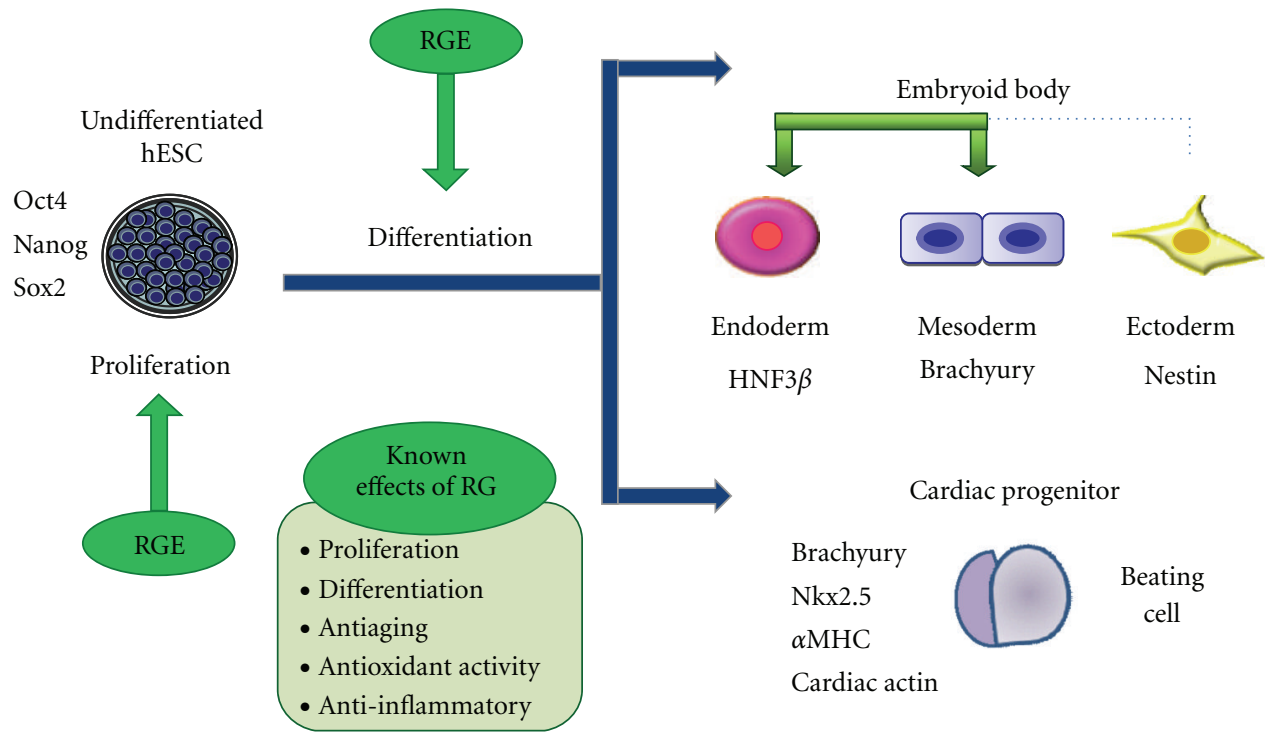

FIGURE 5: Schematic presentation on the various effects of red ginseng extract on the proliferation and differentiation of hESC, embryoid body and hESC-derived cardiac progenitor. hESC: human embryonic stem cells, RGE: red ginseng extract.

the use of ginseng or red ginseng as gene expressionregulating factors in hESCs is extremely rare. To our knowledge, our current paper is the first to observe the facilitating effects of ginseng on the proliferation and differentiation of hESCs. This is important information since the aforementioned beneficial effects have been identified in human stem cells, which provides the basis for further studies to evaluate the long-term effects of RG.

hESCs usually proliferate unlimitedly in vitro without loss of pluripotency [9]. Many paracrine factors such as FGF2 contribute to the maintenance of stemness in hESCs [23-25]. Ginseng is known as a cardiotonic factor for cardiomyocytes [26] and regulator of cardiac contraction [27]. To determine the effects of RG extract, we observed the morphology after RG-treatment, based on the general tendency of hESCs to alternate their morphology after treatment with inducing or soluble factors. RG-treated hESCs showed larger colony size, increased in proliferation and expression of pluripotency markers. RG extract also promoted differentiation into mesendoderm lineage at embryoid body stage, which is evaluated by the increased expression of Brachyury and HNF $3 \beta$. It may imply that ginseng extract could affect the cell differentiation at embryoid body stage. In addition, RG treatment increased the expression of Brachyury, an early mesendodermal marker, at the earlier stage of hESCderived CPs and Nkx2.5, a cardiac-specific marker, at later stage of hESC-derived CPs. These results suggest that RG extracts have diverse effects according to the different stages of undifferentiated hESCs and differentiating cells.

Intriguingly, we observed that different effects of RG were observed at the different stages of undifferentiated hESCs in differentiating cells. RG extract treatment led to an accelerated proliferation of undifferentiated hESCs, and promoted the differentiation of EBs into mesendoderm lineage (Figure 5). Further studies are needed to determine the effects of each RG component or its combinations, which may be able to explain the various effects of RG extract observed in our study.

In conclusion, this pilot study tried to evaluate the effects of RG extracts on the proliferation and differentiation of hESCs during short-term culture in vitro. From our results, RG extracts promoted the proliferation of undifferentiated hESCs, preferentially promoted differentiation of EBs into mesendoderm lineage, and upregulated cardiac-specific expression on hESC-derived cardiac progenitors.

\section{Acknowledgments}

The authors thank Ji Hyun Choi for technical assistance. This work was supported by a grant from the Korean Society of Ginseng funded by the Korea Ginseng Cooperation, a National Research Foundation of Korea Grant funded by the Korean Government (2010-0016819) and Stem Cell Research Center of the 21st Century Frontier Research Program funded by the Ministry of Education, Science, and Technology, Republic of Korea (SC1150).

\section{References}

[1] T. Himi, H. Saito, and N. Nishiyama, "Effect of ginseng saponins on the survival of cerebral cortex neurons in cell cultures," Chemical and Pharmaceutical Bulletin, vol. 37, no. 2, pp. 481-484, 1989.

[2] T.-C. Wen, H. Yoshimura, S. Matsuda, J.-H. Lim, and M. Sakanaka, "Ginseng root prevents learning disability and neuronal loss in gerbils with 5-minute forebrain ischemia," Acta Neuropathologica, vol. 91, no. 1, pp. 15-22, 1996.

[3] S. Sengupta, S.-A. Toh, L. A. Sellers et al., "Modulating angiogenesis: the yin and the yang in ginseng," Circulation, vol. 110, no. 10, pp. 1219-1225, 2004. 
[4] Z.-Q. Liu, X.-Y. Luo, G.-Z. Liu, Y.-P. Chen, Z.-C. Wang, and Y.-X. Sun, "In vitro study of the relationship between the structure of ginsenoside and its antioxidative or prooxidative activity in free radical induced hemolysis of human erythrocytes," Journal of Agricultural and Food Chemistry, vol. 51, no. 9, pp. 2555-2558, 2003.

[5] Y. J. Surh, H. K. Na, J. Y. Lee, and Y. S. Keum, "Molecular mechanisms underlying anti-tumor promoting activities of heat-processed Panax ginseng C.A. Meyer," Journal of Korean medical science, vol. 16, pp. S38-S41, 2001.

[6] D. Kiefer and T. Pantuso, "Panax ginseng," American Family Physician, vol. 68, no. 8, pp. 1539-1542, 2003.

[7] T.-K. Lee, R. M. Johnke, R. R. Allison, K. F. O’Brien, and L. J. Dobbs Jr., "Radioprotective potential of ginseng," Mutagenesis, vol. 20, no. 4, pp. 237-243, 2005.

[8] C. K. B. Ferrari, "Functional foods, herbs and nutraceuticals: towards biochemical mechanisms of healthy aging," Biogerontology, vol. 5, no. 5, pp. 275-289, 2004.

[9] J. A. Thomson, "Embryonic stem cell lines derived from human blastocysts," Science, vol. 282, no. 5391, pp. 1145-1147, 1998.

[10] S. K. Oh, H. S. Kim, H. J. Ahn et al., "Derivation and characterization of new human embryonic stem cell lines: SNUhES1, SNUhES2, and SNUhES3," Stem Cells, vol. 23, no. 2, pp. 211-219, 2005.

[11] M. S. Cho, Y.-E. Lee, J. Y. Kim et al., "Highly efficient and largescale generation of functional dopamine neurons from human embryonic stem cells," Proceedings of the National Academy of Sciences of the United States of America, vol. 105, no. 9, pp. 3392-3397, 2008.

[12] Y. M. Cho, J. M. Lim, D. H. Yoo et al., "Betacellulin and nicotinamide sustain PDX1 expression and induce pancreatic $\beta$-cell differentiation in human embryonic stem cells," Biochemical and Biophysical Research Communications, vol. 366, no. 1, pp. 129-134, 2008.

[13] Y. Y. Kim, S.-Y. Ku, J. Jang et al., "Use of long-term cultured embryoid bodies may enhance cardiomyocyte differentiation by BMP2," Yonsei Medical Journal, vol. 49, no. 5, pp. 819-827, 2008.

[14] S.-L. Wang, Y.-B. Li, Y.-P. Wang, and M. Feng, "Effect of TSPG on proliferation and differentiation of human embryonic neural stem cell into dopaminergic neuron," Zhongguo Zhongyao Zazhi, vol. 32, no. 13, pp. 1310-1313, 2007.

[15] T. Sasaki, K.-B. Oh, H. Matsuoka, and M. Saito, "Effect of Panax ginseng components on the differentiation of mouse embryonic stem cells into cardiac-like cells," Yakugaku Zasshi, vol. 128, no. 3, pp. 461-467, 2008.

[16] S. K. Oh, H. S. Kim, Y. B. Park et al., "Methods for expansion of human embryonic stem cells," Stem Cells, vol. 23, no. 5, pp. 605-609, 2005.

[17] S. Helms, "Cancer prevention and therapeutics: Panax ginseng," Alternative Medicine Review, vol. 9, no. 3, pp. 259-274, 2004.

[18] E. Helmes, V. E. Lewis, and A. Allan, "Australian Lawyers' views on competency issues in older adults," Behavioral Sciences and the Law, vol. 22, no. 6, pp. 823-831, 2004.

[19] A. S. Attele, Y.-P. Zhou, J.-T. Xie et al., "Antidiabetic effects of Panax ginseng berry extract and the identification of an effective component," Diabetes, vol. 51, no. 6, pp. 1851-1858, 2002.

[20] G. Zuo, T. Guan, D. Chen et al., "Total saponins of Panax Ginseng induces K562 cell differentiation by promoting internalization of the erythropoietin receptor," American Journal of Chinese Medicine, vol. 37, no. 4, pp. 747-757, 2009.
[21] D. G. Popovich, S. Y. Yeo, and W. Zhang, "Ginseng (Panax quinquefolius) and Licorice (Glycyrrhiza uralensis) Root Extract Combinations Increase Hepatocarcinoma Cell (HepG2) Viability," Evidence-Based Complementary and Alternative Medicine. In press.

[22] Y. Lei, Q. Gao, and K.-J. Chen, "Effects of extracts from panax notoginseng and panax ginseng fruit on vascular endothelial cell proliferation and migration in vitro," Chinese Journal of Integrative Medicine, vol. 14, no. 1, pp. 37-41, 2008.

[23] M. Amit, C. Shariki, V. Margulets, and J. Itskovitz-Eldor, "Feeder layer- and serum-free culture of human embryonic stem cells," Biology of Reproduction, vol. 70, no. 3, pp. 837-845, 2004.

[24] D. James, A. J. Levine, D. Besser, and A. Hemmati-Brivanlou, "TGF $\beta /$ activin/nodal signaling is necessary for the maintenance of pluripotency in human embryonic stem cells," Development, vol. 132, no. 6, pp. 1273-1282, 2005.

[25] R.-H. Xu, R. M. Peck, D. S. Li, X. Feng, T. Ludwig, and J. A. Thomson, "Basic FGF and suppression of BMP signaling sustain undifferentiated proliferation of human ES cells," Nature Methods, vol. 2, no. 3, pp. 185-190, 2005.

[26] J. Yuan, W. Guo, B. Yang, P. Liu, Q. Wang, and H. Yuan, "116 cases of coronary angina pectoris treated with powder composed of radix ginseng, radix notoginseng and succinum," Journal of Traditional Chinese Medicine, vol. 17, no. 1, pp. 1417, 1997.

[27] G. I. Scott, P. B. Colligan, B. H. Ren, and J. Ren, "Ginsenosides $\mathrm{Rb} 1$ and Re decrease cardiac contraction in adult rat ventricular myocytes: role of nitric oxide," British Journal of Pharmacology, vol. 134, no. 6, pp. 1159-1165, 2001. 


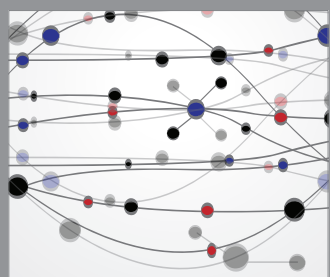

The Scientific World Journal
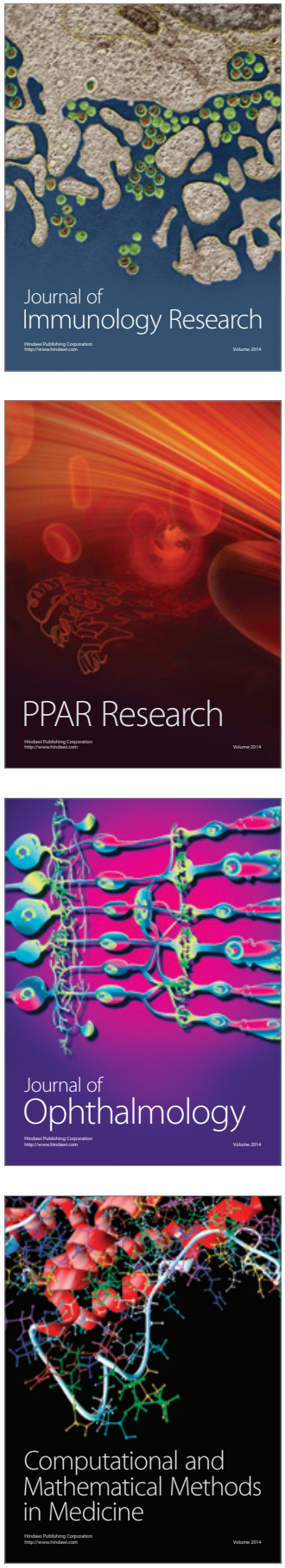

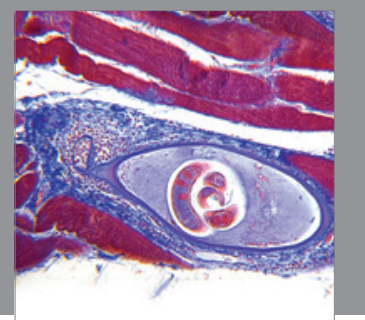

Gastroenterology

Research and Practice
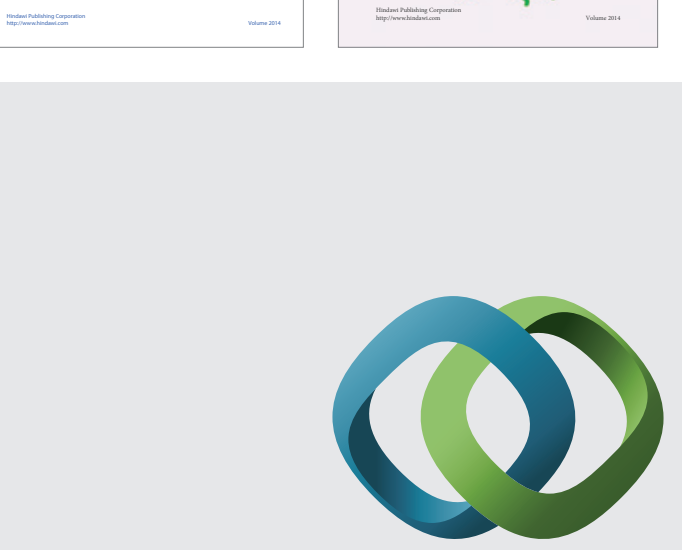

\section{Hindawi}

Submit your manuscripts at

http://www.hindawi.com
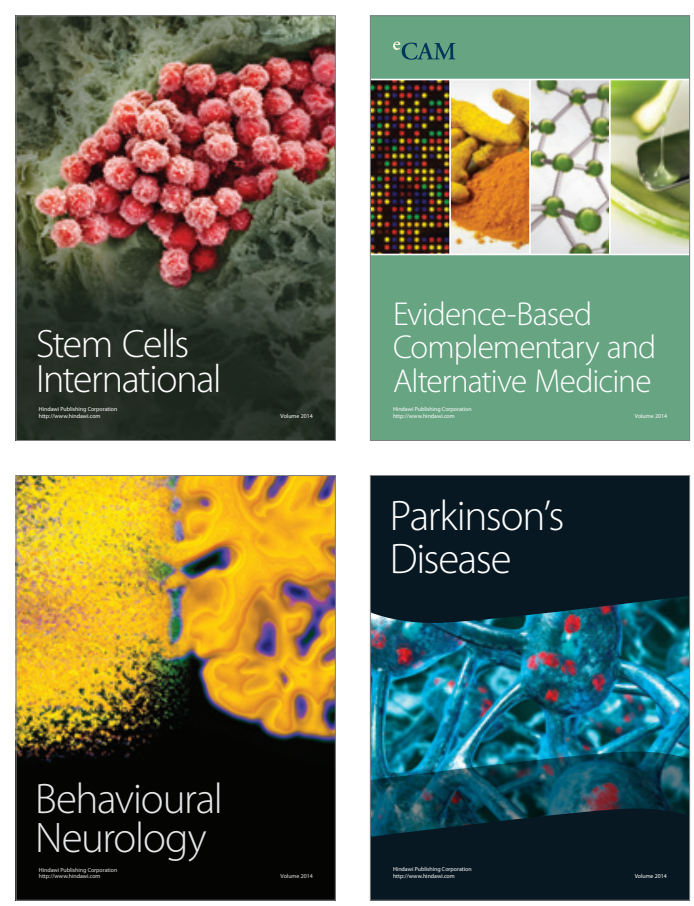

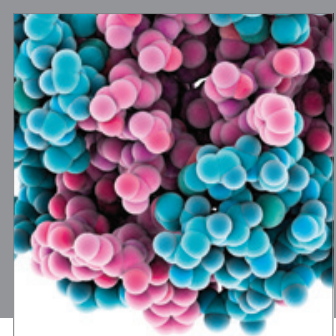

Journal of
Diabetes Research

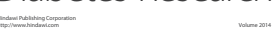

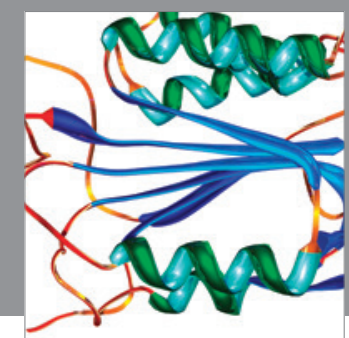

Disease Markers
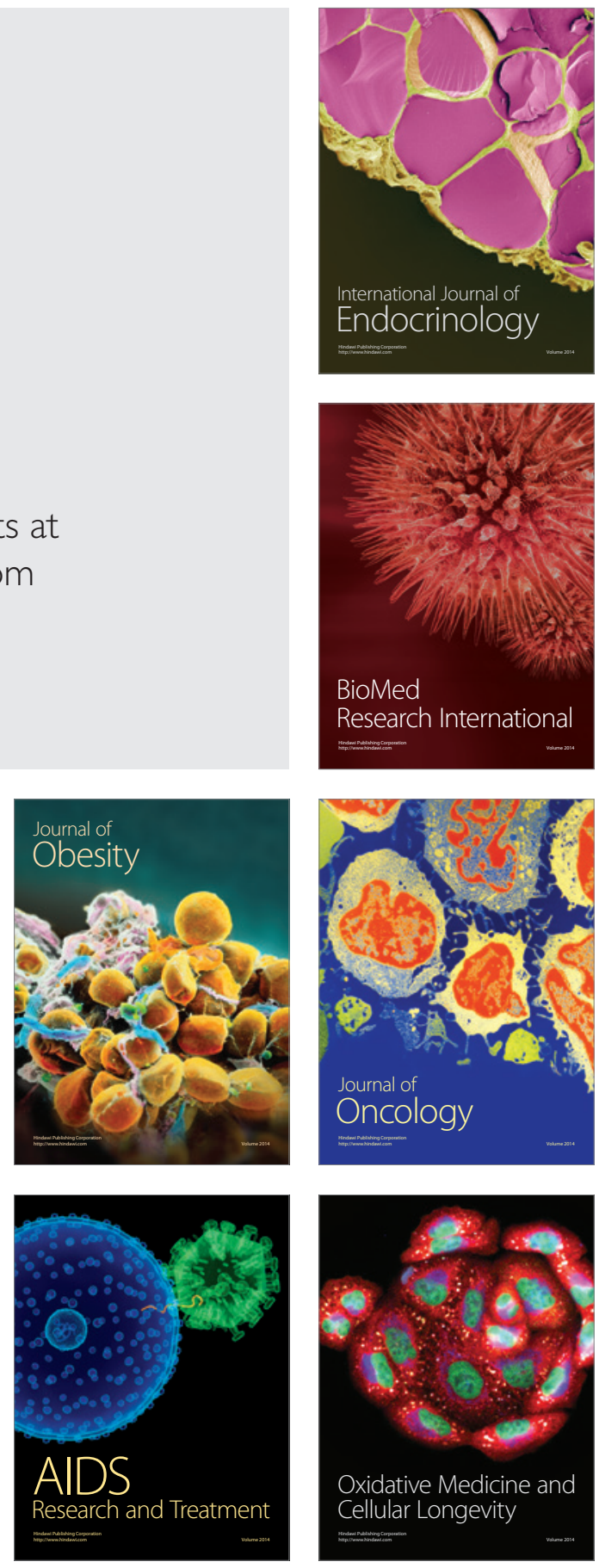